CYSTIC FIBROSIS

\title{
Correlation of forced oscillation technique in preschool children with cystic fibrosis with pulmonary inflammation
}

\author{
S Brennan, G L Hall, F Horak, A Moeller, P M C Pitrez, A Franzmann, S Turner, N de Klerk, \\ P Franklin, K R Winfield, E Balding, S M Stick, P D Sly
}

Thorax 2005;60:159-163. doi: 10.1136/thx.2004.026419

See end of article for authors' affiliations

\section{Correspondence to:} Dr S Brennan, Division of Clinical Sciences, TVW Telethon Institute for Child Health Research, P O Box 855, West Perth, WA 6872, Western Australia; shivs@ichr.uwa.edu.au

Received 7 April 2004 Accepted 27 August 2004
Background: Lung disease in cystic fibrosis (CF) is established in early childhood with recurrent bacterial infections and inflammation. Using spirometry, the effect of this early lung damage cannot be measured until a child is 6 years of age when some irreversible lung damage may already have occurred. Techniques for measurement of lung function in infants and young children include raised volume rapid thoracic compression (RVRTC) and low frequency forced oscillation (LFFOT). The aim of this study was to investigate the role of inflammation and infection on a population of infants and young children with CF and to determine whether lung function in this population (measured by LFFOT) is affected by early lung disease.

Methods: Lung function was measured by LFFOT in 24 children undergoing bronchoalveolar lavage (BAL) on 27 occasions as part of an annual programme while still under general anaesthesia. Following lung function testing, three aliquots of saline were instilled into the right middle or lower lobe. The first aliquot retrieved was processed for the detection of microbes, and the remaining aliquots were pooled to assess inflammatory markers (cytology, IL-8, NE, LTB 4 ).

Results: Inflammation (percentage and number of neutrophils) was significantly higher in children with infections $(p<0.001, p=0.04$, respectively), but not in those with symptoms. Several markers of inflammation significantly correlated with LFFOT parameters (R, $G$, and $\eta)$.

Conclusion: Infections and inflammation are established before symptoms are apparent. Inflammation is correlated with measures of parenchymal changes in lung function measured by LFFOT.
L ung disease in cystic fibrosis (CF) begins in early childhood and is characterised by the presence of - persistent infections and inflammation, even in the absence of clinically apparent disease. ${ }^{12}$ Measures of cellular burden and soluble chemotactic factors such as interleukin-8 (IL-8) and leukotriene $\mathrm{B}_{4}\left(\mathrm{LTB}_{4}\right)$ are increased in the plasma, ${ }^{3}$ sputum, ${ }^{3}{ }^{4}$ breath condensates, ${ }^{5}$ and broncholaveolar lavage (BAL) fluid ${ }^{6}$ of patients with CF compared with controls. The impact of this early inflammation on lung function in early childhood is not fully known.

Lung function measurements (forced expiratory volume in l second $\left(\mathrm{FEV}_{1}\right)$, forced vital capacity $\left.(\mathrm{FVC})\right)$ are gold standard measures that can be reliably used in older children and adults with CF, both clinically and as outcome measures in therapeutic trials. The use of BAL in preschool children has shown the value of early assessment of infections and inflammatory markers, demonstrating that the disease process begins before lung disease is apparent clinically. ${ }^{2}{ }^{8}$ Careful measurements of pulmonary function in infants and young children with CF also show detectable abnormalities early in the clinical course. ${ }^{9-13}$ Two recent studies have compared inflammatory indices with lung function measures taken concurrently. ${ }^{10}{ }^{12}$ Nixon and colleagues found lower lung function, as measured by the raised volume rapid thoracic compression technique (RVRTC), in those with symptomatic lung disease and with the presence of infection detected in BAL fluid. ${ }^{10}$ Lung function did not appear to be related to inflammation per se. In contrast, Dakin and coworkers identified significant relationships between specific respiratory system compliance (sCRS), the pathogen load, and the number of neutrophils present in BAL fluid.

None of the previous studies has used a technique that is capable of providing separate estimates of the mechanical properties of the airway and pulmonary parenchyma. Lung disease in CF begins in the distal airways and should therefore be reflected in abnormalities of the intra-parenchymal airways and parenchymal mechanics. The low frequency forced oscillation technique (LFFOT) allows the measurement of the respiratory system impedance (Zrs) at a range of frequencies and allows partitioning of lung function into components representing the airways and pulmonary parenchyma. We have previously shown that the LFFOT can be used to make such measurements in infants, ${ }^{14}$ and that infants and young children with a history of recurrent wheeze persisting into the second year of life have abnormalities in both parenchymal and airway mechanics. ${ }^{15}$ This technique is therefore suitable for examining early lung disease in young children with CF and for investigating the relationship between inflammation, infection, and lung function in such infants. The central hypothesis tested in this study was that the presence of neutrophilic inflammation and infection detected in BAL fluid is associated with alterations in lung function, as measured by LFFOT.

\section{METHODS}

\section{Subjects}

In the period 1997-2002 all children under 6 years of age were given the opportunity to take part in the clinical BAL programme for detecting the presence of infection provided by the CF clinic at Princess Margaret Hospital for Children,

\footnotetext{
Abbreviations: BAL, bronchoalveolar lavage; $C F$, cystic fibrosis; $\eta$, hysteresivity (ratio of damping to elastance); $\mathrm{FEV}_{1}$, forced expiratory volume in 1 second; FVC, forced vital capacity; Grs, tissue damping; Hrs, tissue elastance; IAW, inertance; IL, interleukin; LFFOT, low frequency forced oscillation technique; $\mathrm{LTB}_{4}$, leukotriene $\mathrm{B}_{4} ;$ MOF, mixed oral flora; $N E$, neutrophil elastase; Prs, transrespiratory pressure; Raw, tissue resistance; sCRS, specific respiratory system compliance; TCC, total cell count; Zrs, respiratory system impedance
} 
Perth, Australia. BAL was performed in newly diagnosed children with CF as part of the clinical assessment and repeated annually. Parents were asked for permission for measurement of lung function following BAL, and for use of surplus BAL fluid for assessment of inflammation. The diagnosis of CF was confirmed by a sweat chloride level of $\geqslant 60 \mathrm{mmol} / \mathrm{I}^{16}$ and gene mutation analysis, screening initially for four common mutations and up to 270 mutations for unknown compound heterozygotes identified.

The study was approved by the ethics committee at Princess Margaret Hospital, Perth and written consent was obtained from the parents of all subjects.

\section{Bronchoalveolar lavage}

Bronchoscopy and lavage were conducted under general anaesthesia using sevofluorane for induction and intravenous propofol for maintenance. Three aliquots of normal saline $(1 \mathrm{ml} / \mathrm{kg})$ were instilled into the right middle lobe or right lower lobe and retrieved using low pressure suction. The first aliquot was sent to the Department of Microbiology for identification of bacteria, fungal elements, and viruses. The remaining aliquots were pooled and stored on ice until processed (within 3 hours) for the assessment of inflammation. The median retrieval rate from the second and third aliquots was $22 \%$ of fluid instilled.

Symptoms present at the time of BAL were recorded. Patients were classified as symptomatic if they had any current cough, wheeze, or rhinitis.

\section{Microbiology}

BAL samples were cultured on blood, CLED, Fildes' agar and Saraboud's agar with chloramphenicol. Viruses (RSV, parainfluenza 1, 2 and 3, influenza A and B, adenovirus, and cytomegalovirus) were detected using direct immunofluorescence and/or rapid viral tissue culture. Significant microbial "infection" was considered as $>10^{4} \mathrm{cfu} / \mathrm{ml}$. Bacterial densities of $10^{2}-10^{4} \mathrm{cfu} / \mathrm{ml}$ were recorded as isolated colonies. Mixed oral flora (MOF) was not considered a significant pathogen but was recorded. BAL with MOF or isolated colonies recorded was classified as "uninfected" for the purposes of this study.

\section{Inflammation}

The BAL fluid was centrifuged ( $167 g$ for 5 minutes) and the supernatant decanted, aliquoted, and frozen at $-80^{\circ} \mathrm{C}$ until subsequent analysis. Cell pellets were resuspended and total cell counts (TCC) conducted. Cells resuspended to $10^{6}$ cells/ $\mathrm{ml}$ for cytospins were stained with Leishman's. A blinded observer then conducted a differential cell count of 300 cells.

Interleukin-8 was measured using a commercially available ELISA (Biosource, USA) with a detection limit of $20 \mathrm{pg} /$ ml. Neutrophil elastase (NE) activity was measured by a modified enzymatic assay ${ }^{17}$ using n-succinyl Tri-ala as the specific substrate with a lower limit of $1.6 \mu \mathrm{g} / \mathrm{ml}$. LTB $_{4}$ was measured using an enzyme immunoassay kit (AmershamPharmacia, USA) with a detection limit of $0.31 \mathrm{pg} / \mathrm{ml}$.

\section{Lung function}

Lung function was measured by LFFOT under general anaesthesia and immediately before BAL was performed on 27 occasions in 24 children (three children were measured twice).

\section{LFFOT measurement apparatus and analysis}

The respiratory system impedance (Zrs) was measured using the LFFOT as previously described in infants and young children. ${ }^{14} 151819$ Briefly, the child's lungs were inflated to a transrespiratory pressure (Prs) of $20 \mathrm{~cm} \mathrm{H}_{2} \mathrm{O}$ three times.
Table 1 Details of children at time of first BAL and of the whole group

\begin{tabular}{|c|c|c|}
\hline Parameters & $\begin{array}{l}\text { First visit } \\
(N=24)\end{array}$ & $\begin{array}{l}\text { Total visits } \\
(\mathrm{N}=27)\end{array}$ \\
\hline No of subjects & 24 & 24 \\
\hline $\begin{array}{l}\text { Median (IQR) age at } \\
\text { first study }\end{array}$ & $1.58(1.07-2.75)$ & $1.58(1.12-2.77)$ \\
\hline Sex & $12 \mathrm{M}(50 \%)$ & $14 \mathrm{M}(52 \%)$ \\
\hline \multicolumn{3}{|l|}{ Genotype } \\
\hline Homozygote & $17(70.8 \%)$ & 19 (70.3\%) \\
\hline Heterozygote & 7 & 8 \\
\hline $\begin{array}{l}\text { Compound heterozygote } \\
\text { (not F508) }\end{array}$ & 1 & 1 \\
\hline No of visits & 27 & 27 \\
\hline $\begin{array}{l}\text { No of children with } \\
\text { repeat visits }\end{array}$ & 3 & 3 \\
\hline \multicolumn{3}{|l|}{ Microbiology at time of visit } \\
\hline No pathogen & $12(48 \%)$ & $13(48 \%)$ \\
\hline Mixed oral flora & $3(12 \%)$ & $3(12 \%)$ \\
\hline A fumigatus & $1(4 \%)$ & $1(4 \%)$ \\
\hline$S$ aureus & $3(12 \%)$ & $3(12 \%)$ \\
\hline$H$ influenzae & $1(4 \%)$ & $2(8 \%)$ \\
\hline$P$ aeruginosa & $4(16.7 \%)$ & $4(15 \%)$ \\
\hline Enterobacter sp & $2(8 \%)$ & $2(8 \%)$ \\
\hline Calbicans & $1(4 \%)$ & $1(4 \%)$ \\
\hline Other & $3(12 \%)$ & $3(12 \%)$ \\
\hline \multicolumn{3}{|l|}{ Treatments at time of BAL } \\
\hline No antibiotics & & $6(22.2 \%)$ \\
\hline Amoxycillin/clavulanate & & $18(66.7 \%)$ \\
\hline $\mathrm{C} / \mathrm{T} / \mathrm{S}$ & & $3(11.1 \%)$ \\
\hline
\end{tabular}

Following the final inflation the airway was occluded at the raised Prs inducing the Hering-Breur reflex. During the resulting pause in breathing a broadband forcing signal (0.5$20 \mathrm{~Hz}$ ) was applied to the airway opening via a soft rimmed face mask (Kings Systems, USA). Airway opening pressure (Pao) and flow (V'ao) were measured and the Zrs calculated by transforming the Pao and V'ao signals into the frequency domain by using a fast Fourier transformation algorithm. Zrs was then calculated as the ratio of pressure and flow at each frequency contained in the forcing function. Three to five Zrs acceptable spectra were obtained from each subject.

After omitting any data points corrupted by cardiac artefacts, the four parameter constant phase model ${ }^{20}$ was fitted to the individual spectra and the respective components averaged using the following formula:

$$
\mathrm{Zrs}=\mathrm{Raw}+j \omega \mathrm{Iaw}+(\mathrm{Grs}-j \mathrm{Hrs}) / \omega^{\alpha}
$$

where Raw and Iaw are the frequency independent resistance and inertance, respectively, attributed to the airways; Grs and Hrs are the coefficients of tissue damping and elastance, respectively; $j$ is the imaginary unit, $\omega$ the angular frequency, and $\alpha$ describes the frequency dependence of the impedence of the pulmonary parenchyma. Hysteresivity $(\eta)$, which describes the coupling of the elastic and dissipative forces of the parenchyma, ${ }^{21}$ was calculated as the ratio of Grs and Hrs $(\eta=\mathrm{Grs} / \mathrm{Hrs})$.

\section{Statistical analysis}

The relationships between lung function parameters, inflammatory parameters, infection, and symptoms were investigated using generalised estimating equations (GEE) using Stata statistical software (StataCorp, Texas, USA) which allowed for repeat measures in subjects. Body length was included as an independent variable in these equations for all tests except for hysteresivity $(\eta)$ which is a ratio of Grs to Hrs For these tests the $\log _{10}$ of TCC, neutrophils per ml, IL-8, and $\mathrm{LTB}_{4}$ was used to correct for a skewed distribution in these measures. Values quoted are median values with 25 th-75th 
Table 2 Lung function and inflammatory parameters in BAL fluid of subjects with and without infection

\begin{tabular}{llll}
\hline & $\begin{array}{l}\text { Uninfected } \\
\mathbf{N}=15 \text { children, } \\
\mathrm{N}=17 \text { visits) }\end{array}$ & $\begin{array}{l}\text { Infectedt } \\
\mathbf{N}=9 \text { children, } \\
\mathrm{N}=10 \text { visits })\end{array}$ & p value \\
\hline Parameter & $1.58(1.12$ to 2.91$)$ & $1.55(1.11$ to 2.51$)$ & $\mathrm{NS}$ \\
$\mathrm{TCC} / \mathrm{ml}\left(\times 10^{6}\right)$ & $0.34(0.23$ to 0.47$)$ & $1.29(0.27$ to 6.01$)$ & 0.098 \\
Neutrophils $(\%)$ & $17.3(8.75$ to 29.10$)$ & $52.0(37.85,81.00)$ & $<0.001^{*}$ \\
Neutrophils/ml $\left(\times 10^{3}\right)$ & $60.03(38.92$ to 91.34$)$ & $667.58(57.52$ to 5341.48$)$ & $0.04^{*}$ \\
IL-8 $(\mathrm{ng} / \mathrm{ml})$ & $0.36(0.18$ to 1.42$)$ & $1.36(0.16$ to 1.91$)$ & $\mathrm{NS}$ \\
$\mathrm{LTB}_{4}(\mathrm{pg} / \mathrm{ml})$ & $6.23(2.4$ to 12.92$)$ & $6.53(4.97$ to 25.88$)$ & $\mathrm{NS}$ \\
Symptomatic $(\%$ visits $)$ & $17 \%$ & $80 \%$ & $\mathrm{NS}$ \\
Raw & $14.32(9.86$ to 20.81$)$ & $12.85(10.03$ to 22.80$)$ & $\mathrm{NS}$ \\
Grs & $27.18(16.8$ to 38.3$)$ & $38.26(25.87$ to 44.13$)$ & $\mathrm{NS}$ \\
Hrs & $115.3(77.4$ to 144.24$)$ & $142.78(110.64$ to 175.82$)$ & $\mathrm{NS}$ \\
$\eta$ & $0.26(0.19$ to 0.32$)$ & $0.25(0.14$ to 0.31$)$ & $\mathrm{NS}$ \\
\hline
\end{tabular}

TCC, total cell count; IL, interleukin; $\mathrm{LTB}_{4}$, leukotriene $\mathrm{B}_{4}$; Raw, tissue resistance; Grs, tissue damping; Hrs, tissue elastance; $\eta$, ratio of damping to elastance.

Values are median (IQR).

${ }^{*} \mathrm{p}<0.05$.

tInfection was categorised as $>10^{4} \mathrm{cfu} / \mathrm{ml}$.

Lung function data corrected for body length and number of visits.

interquartile ranges (IQR) in parentheses. p values of $\leqslant 0.05$ were considered significant.

\section{RESULTS}

\section{Subjects}

Twenty four children (50\% boys) with CF successfully underwent BAL and LFFOT during the study period on 27 occasions (three children had one repeat BAL visit). The median (IQR) age of the children at the time of the first BAL was 1.58 years (1.07-2.77) and for all visits was 1.58 years (1.12-2.77). The demographic details of the group including genotype are shown in table 1. Most of the children had either mixed oral flora or no pathogen detected at the time of BAL; $16.7 \%$ had Pseudomonas aeruginosa isolated at the time of first lavage. $77 \%$ of subjects were on antibiotics at the time of BAL with $66.7 \%$ on amoxicillin/clavulanate. No subjects were receiving inhaled antibiotics at the time of BAL (table 1).

\section{Inflammation and infection}

Inflammation was evident in almost all subjects. IL-8 levels in BAL fluid correlated with the total cell burden $\left(r^{2}=0.676\right.$, $\mathrm{p}<0.001$ ) and the number of neutrophils present in BAL fluid $\left(r^{2}=0.729, \mathrm{p}<0.001\right.$; table 2$)$. Increases in TCC $/ \mathrm{ml}$ and both number and percentage of neutrophils were associated with increasing bacterial load ( $p=0.0124, p<0.001$, and $p<0.001$, respectively). There was no significant difference between the children with no growth $(n=13)$ and those with either isolated colonies or MOF $(n=4)$. NE was detected in the BAL fluid from only three of the 27 visits, all of which came from children with an infection.

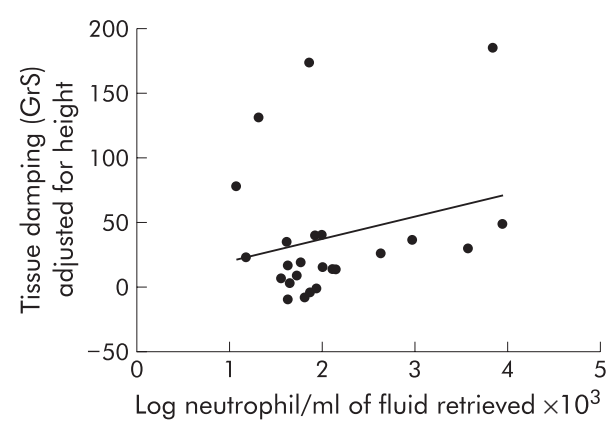

Figure 1 Relationship between tissue damping adjusted for height using linear regression versus the log number of neutrophils per $\mathrm{ml}$ retrieved BAL fluid $\left(\times 10^{3}\right)$ in 24 subjects on 27 occasions.

There was a significant difference in both the percentage and the number of neutrophils in the BAL fluid of symptomatic and asymptomatic children (table 3) but no significant difference in the measures of TCC/ml, IL-8, $\mathrm{LTB}_{4}$, or NE.

\section{Relationship between forced oscillation measurements and markers of inflammation and infection}

Tissue mechanics

After accounting for body length there was a significant correlation between tissue damping and $\mathrm{LTB}_{4}(\mathrm{p}=0.014)$ and IL-8 $(p=0.024)$; there were further significant relationships

Table 3 Inflammation parameters from BAL fluid of subjects who were asymptomatic or symptomatic at the time of BAL

\begin{tabular}{llll}
\hline Parameter & $\begin{array}{l}\text { Asymptomatic } \\
\text { (N=14 children, } \\
\mathrm{N}=16 \text { visits) }\end{array}$ & $\begin{array}{l}\text { Symptomatic } \\
\text { (N=11 children, } \\
\mathrm{N}=11 \text { visits) }\end{array}$ & p value \\
\hline $\mathrm{TCC} / \mathrm{ml}$ & $0.30(0.26$ to 0.42$)$ & $1.29(0.37$ to 6.01$)$ & 0.062 \\
Neutrophils $(\%)$ & $16.5(10.5$ to 26.5$)$ & $53(49.67$ to 80.5$)$ & $<0.001^{*}$ \\
Neutrophils $/ \mathrm{ml}\left(\times 10^{3}\right)$ & $60.03(38.45$ to 90.55$)$ & $667.58(64.3$ to 5341.48$)$ & $0.002^{*}$ \\
IL-8 $(\mathrm{ng} / \mathrm{ml})$ & $0.6(0.23$ to 1.48$)$ & $1.13(0.16$ to 1.87$)$ & $\mathrm{NS}$ \\
$\mathrm{LTB}_{4}(\mathrm{pg} / \mathrm{ml})$ & $5.46(3.16$ to 9.15$)$ & $7.87(4.47$ to 26.67$)$ & $\mathrm{NS}$ \\
\hline
\end{tabular}

TCC, total cell count; IL, interleukin; $\mathrm{LTB}_{4}$, leukotriene $\mathrm{B}_{4}$ Values are median (IQR) ${ }^{*} \mathrm{p}<0.05$. 


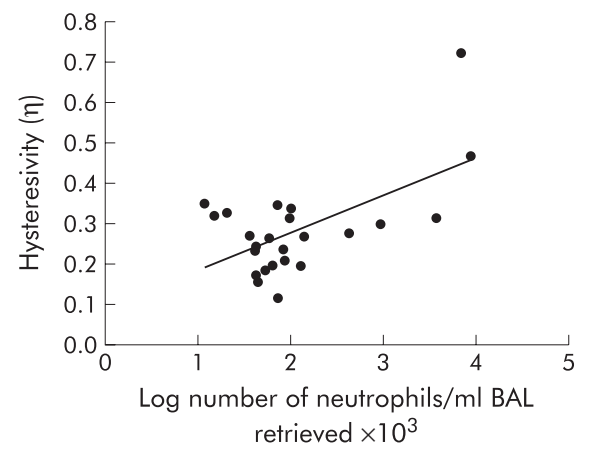

Figure 2 Relationship between hysteresivity and the log number of neutrophils per $\mathrm{ml}$ retrieved BAL fluid $\left(\times 10^{3}\right)$ in 24 subjects on 27 occasions.

between Grs and markers of total cell burden, neutrophil number, and TCC/ml fluid retrieved $(\mathrm{p}=0.009, \mathrm{p}=0.013$; fig 1). Tissue hysteresivity was also significantly related to TCC $(\mathrm{p}<0.001)$ and neutrophil number $(\mathrm{p}<0.001$, fig 2$)$ as well as IL-8 in BAL fluid ( $p=0.001)$. Tissue elastance had no significant relationship with any of the inflammatory parameters measured (table 4).

\section{Airway mechanics}

The resistance of the airways (Raw) was significantly correlated only with the perecentage of neutrophils in the BAL fluid $(p=0.036)$. It was not related to any other markers of neutrophilic inflammation measured.

Ten of the children were infected with respiratory pathogens at the time of testing. There was a significant difference between the infected and uninfected subjects in both the percentage $(\mathrm{p}<0.001)$ and the number of neutrophils $(p=0.04)$ in BAL fluid but not in any of the soluble markers. There was no significant difference between the measures of lung function (airway or parenchymal) in infected or uninfected children.

Table 4 Relationships between inflammatory markers and measures of lung function assessed by the low frequency forced oscillation technique (LFFOT)

\begin{tabular}{|c|c|c|}
\hline Descriptor & $Z$ value & $p$ value \\
\hline \multirow[t]{4}{*}{$\%$ neutrophils $v$ LFFOT } & Raw $=2.09$ & $0.036 *$ \\
\hline & $\mathrm{Grs}=2.35$ & 0.19 \\
\hline & $\mathrm{Hrs}=0.41$ & 0.68 \\
\hline & $\eta=3.03$ & $0.002^{*}$ \\
\hline $\log _{10}$ no of neutrophils $v$ & Raw $=1.90$ & 0.057 \\
\hline \multirow{3}{*}{ LFFOT } & Grs $=2.62$ & $0.009^{*}$ \\
\hline & $\mathrm{Hrs}=0.66$ & 0.955 \\
\hline & $\eta=3.63$ & $<0.001^{*}$ \\
\hline \multirow{4}{*}{$\log _{10}$ TCC $v$ LFFOT } & Raw $=1.72$ & 0.085 \\
\hline & $\mathrm{Grs}=2.47$ & $0.013^{*}$ \\
\hline & $\mathrm{Hrs}=0.65$ & 0.518 \\
\hline & $\eta=3.51$ & $<0.001^{*}$ \\
\hline \multirow[t]{4}{*}{ Log10 IL-8 v LFFOT } & Raw $=1.58$ & 0.115 \\
\hline & Grs $=2.43$ & $0.024^{*}$ \\
\hline & $\mathrm{Hrs}=0.06$ & 0.955 \\
\hline & $\eta=3.31$ & $0.001^{*}$ \\
\hline \multirow{4}{*}{ Log10 LTB $_{4} v$ LFFOT } & Raw $=1.59$ & 0.112 \\
\hline & $\mathrm{Grs}=2.44$ & $0.014^{*}$ \\
\hline & $\mathrm{Hrs}=1.91$ & 0.056 \\
\hline & $\eta=1.54$ & 0.122 \\
\hline \multicolumn{3}{|c|}{$\begin{array}{l}\text { Raw, tissue resistance; Grs, tissue damping; Hrs, tissue elastance; } \eta \text {, ratic } \\
\text { of damping to elastance. } \\
{ }^{*} p<0.05 \text {. }\end{array}$} \\
\hline
\end{tabular}

Relationship between forced oscillation lung function measurements and symptoms

Eleven of the children ( $40.7 \%$ of visits) were symptomatic at the time of testing. No significant differences were detected between asymptomatic and symptomatic subjects in any of the lung function parameters measured by forced oscillation (table 3).

\section{DISCUSSION}

The results of this study show that abnormal lung function is present early in the course of CF and that the LFFOT is sensitive at detecting early lung disease, as defined by the presence of inflammation in BAL fluid.

It is now accepted that lung disease in CF begins in early childhood and, once infections become established, chronic neutrophil driven inflammation contributes to the lung tissue damage and propagates the cycle of inflammation. This is supported by the increase in relevant markers of neutrophilic inflammation in this young population, with significant correlations between the soluble mediator (IL-8) and total cell burden (TCC/ml, neutrophils $/ \mathrm{ml}$ ) suggesting ongoing signalling for further cell recruitment. Once structural changes occur they appear to be irreversible..$^{22}$ It is therefore imperative, if treatments are ever to be tailored to prevent this lung damage, that we find new techniques capable of detecting early changes in lung function associated with the initiation of inflammatory lung disease.

Lung function, measured by spirometry, is insensitive to early structural damage and there is a lag between detecting established infection/inflammation and seeing significant measurable effects on standard lung function tests. Several research groups have worked in past decades to establish methods for measuring parameters of lung function in children too young to conduct spirometric tests reliably including RVRTC, full body plethysmography, and LFFOT. Each of these techniques has been validated in different patient groups and some studies have been used to identify differences in a pre-school CF population ${ }^{13}$ compared with a non-CF control population, while others have tried to identify relationships between inflammation/infection and lung function parameters. ${ }^{10}$

The LFFOT was adapted for use in infants in $1996^{14}$ and has been used to study the normal growth and development of the respiratory system ${ }^{19}$ and to study the site of lung disease in children with recurrent wheeze persisting into the second year of life. ${ }^{15}$ The technique measures input impedance by applying a pressure waveform (forcing function) to the airway opening and measuring the resultant flow produced at the airway opening. The resulting impedance reflects the complex viscoelastic resistance of the respiratory system. The "constant phase" model ${ }^{20}$ has provided an adequate description of the lungs in several species. This model consists of an airway component, represented by a frequency independent airway resistance and airway inertance, and a constant phase tissue component $(\mathrm{G}-j \mathrm{H}) / \omega^{\alpha}$, where $\mathrm{G}$ and $\mathrm{H}$ are coefficients for tissue damping and elastance respectively, $\omega$ is angular frequency, and $\alpha$ describes the frequency dependence of the real (pressure change in phase with flow) and imaginary (pressure change in phase with volume) parts of the impedance. In this context, the airways represent those parts of the respiratory tree where gas moves by convective or bulk flow-that is, from the airway opening to the terminal bronchioles. Those elements of the respiratory tree in which gas diffusion predominates (respiratory bronchioles, alveolar ducts, alveoli, and interstitium) are represented in the pulmonary component. The accuracy of the partitioning of lung function by the constant phase model into components representing the airways and pulmonary parenchyma has been demonstrated in animal models. ${ }^{23}$ 
As lung disease in CF is now believed to begin in the peripheral lung, one would expect that measurement techniques sensitive to changes in the mechanical properties of the intraparenchymal airways and the lung parenchyma would detect lung disease earlier than those that represent both the airways and lung parenchymal function as a single global number. Fitting the constant phase model to impedance spectra obtained by the LFFOT provides an assessment of the peripheral lung. The data from the present study have shown consistent significant relationships between parenchymal mechanics (tissue damping and hysteresivity) and markers of neutrophil driven inflammation-total cell burden, neutrophils (number and percentage), and IL-8. This finding supports the data of Dakin et al ${ }^{12}$ who found a significant relationship between the percentage of neutrophils and specific compliance measured by the single breath occlusion technique. They measured specific compliance, which reflects both the compliance of the respiratory system (the lungs and chest wall) and the resistive properties of the pulmonary parenchyma. Unlike Dakin et $a l,{ }^{12}$ we did not find any differences in lung function between children classed as symptomatic and those classed as asymptomatic. As all of our studies were performed as part of an elective BAL programme, children were only scheduled for study when they were clinically well, so we cannot comment directly on changes in lung function during periods of increased symptoms such as that which occurs during a respiratory exacerbation. The children classed as symptomatic had only slightly more pulmonary inflammation than asymptomatic subjects. Symptoms were recorded at the time of BAL in a generalised fashion (clinically well, presence of cough, rhinitis, etc) without the use of any objective tool. No such tool/questionnaire is currently available and validated for a preschool population. Such a tool would have provided a valuable reference for objective collection of "symptomatic" data.

Children with detectable infection (defined as $>10^{4} \mathrm{cfu}$ / $\mathrm{ml}$ ) had significantly more inflammation than uninfected subjects, but these differences were not associated with significant differences in the lung function parameters measured. We cannot therefore determine with certainty whether the level of infection and inflammation measured was insufficient to affect lung function noticeably or whether the techniques we used were not sensitive enough to detect the very early changes. Given the mild disease of the children with "infection", the power of this study was insufficient to detect changes in lung function between infected and uninfected children. A larger study cohort will be required to investigate this fully.

The subjects in our study, with a median (range) age of 1.58 (1.07-2.75) years, represent a population with very early stages of lung disease as indicated by the level of inflammation which was considerably less than that measured by both Dakin et $a l^{12}$ and Nixon et al. ${ }^{10}$ With this in mind, it is encouraging to find that lung function measured with the LFFOT did correlate with markers of neutrophil driven inflammation, even in children who had no detectable infection or were clinically asymptomatic.

In conclusion, we have shown that measurements of lung function that provide separate estimates of the mechanical properties of the airways and pulmonary parenchyma can be collected in a CF population and that, in particular, estimates of parenchymal hysteresivity and tissue damping significantly correlate with pulmonary inflammation detected by BAL. Measurements made with LFFOT may therefore hold promise for detecting lung disease early in the clinical course of young children with CF.

\section{Authors' affiliations}

S Brennan, P M C Pitrez, A Franzmann, N de Klerk, K R Winfield, S M Stick, P D Sly, Telethon Institute for Child Health Research, Perth, Western Australia

S Brennan, P M C Pitrez, A Franzmann, N de Klerk, K R Winfield,

S M Stick, P D Sly, Centre for Child Health Research, University of Western Australia, Perth, Western Australia

G L Hall, F Horak, A Moeller, P M C Pitrez, A Franzmann, S Turner, P Franklin, E Balding, S M Stick, P D Sly, Department of Respiratory Medicine, Princess Margaret Hospital for Children, Perth, Western Australia

G L Hall, P Franklin, School of Paediatric and Child Health, University of Western Australia, Perth, Western Australia

This work has been financially supported by the National Health and Medical Research Council of Australia, Australian Cystic Fibrosis Research Trust and Telethon, Western Australia.

\section{REFERENCES}

1 Balough K, McCubbin M, Weinberger $M$, et al. The relationship between infection and inflammation in the early stages of lung disease from cystic fibrosis. Pediatr Pulmonol 1995;20:63-70.

2 Khan TZ, Wagener JS, Bost T, et al. Early pulmonary inflammation in infants with cystic fibrosis. Am J Respir Crit Care Med 1995;151:1075-82.

3 Wyatt HA, Sampson AP, Balfour-Lynn IM, et al. Production of the potent neutrophil chemokine, growth-related protein alpha (GROalpha), is not elevated in cystic fibrosis children. Respir Med 2000;94:106-11.

4 Dean TP, Dai Y, Shute JK, et al. Interleukin-8 concentrations are elevated in bronchoalveolar lavage, sputum, and sera of children with cystic fibrosis. Pediatr Res 1993;34:159-61.

5 Carpagnano GE, Barnes PJ, Geddes DM, et al. Increased leukotriene B4 and interleukin-6 in exhaled breath condensate in cystic fibrosis. Am J Respir Crit Care Med 2003; 167:1109-12.

6 Konstan MW, Walenga RW, Hilliard KA, et al. Leukotriene B4 markedly elevated in the epithelial lining fluid of patients with cystic fibrosis. Am Rev Respir Dis 1993;148:896-901.

7 Muhlebach MS, Stewart PW, Leigh MW, et al. Quantitation of inflammatory responses to bacteria in young cystic fibrosis and control patients. Am J Respir Crit Care Med 1999; 160:186-91.

8 Armstrong D, Grimwood K, Carzino R, et al. Lower respiratory infection and inflammation in infants with newly diagnosed cystic fibrosis. BMJ 1995;310:1571-2.

9 Turner DJ, Lanteri CJ, LeSouef PN, et al. Improved detection of abnormal respiratory function using forced expiration from raised lung volume in infants with cystic fibrosis. Eur Respir J 1994;7:1995-9.

10 Nixon GM, Armstrong DS, Carzino R, et al. Early airway infection, inflammation, and lung function in cystic fibrosis. Arch Dis Child 2002;87:306-11.

11 Gappa M, Ranganathan SC, Stocks J. Lung function testing in infants with cystic fibrosis: lessons from the past and future directions. Pediatr Pulmonol 2001;32:228-45.

12 Dakin $\mathrm{CJ}$, Numa $\mathrm{AH}$, Wang $\mathrm{H}$, et al. Inflammation, infection, and pulmonary function in infants and young children with cystic fibrosis. Am J Respir Crit Care Med 2002; 165:904-10.

13 Ranganathan SC, Bush A, Dezateux C, et al. Relative ability of full and partial forced expiratory maneuvers to identify diminished airway function in infants with cystic fibrosis. Am J Respir Crit Care Med 2002;166:1350-7.

14 Sly PD, Hayden MJ, Petak F, et al. Measurement of low-frequency respiratory impedance in infants. Am J Respir Crit Care Med 1996;154:161-6.

15 Hall GL, Hantos Z, Sly PD. Altered respiratory tissue mechanics in asymptomatic wheezy infants. Am J Respir Crit Care Med 2001; 164:1387-91

16 Gibson LE, Cooke RE. A test for concentration of electrolytes in sweat in cystic fibrosis of the pancreas utlizing pilocarpine by iontophoresis. Pediatrics 1959;23:545.

17 Bieth J, Spiess B, Wermuth CG. The synthesis and analytical use of a highly sensitive and convenient substrate of elastase. Biochem Med 1974;11:350-7.

18 Hayden MJ, Petak F, Hantos Z, et al. Using low-frequency oscillation to detect bronchodilator responsiveness in infants. Am J Respir Crit Care Med 1998; 157:574-9.

19 Hall GL, Hantos Z, Petak F, et al. Airway and respiratory tissue mechanics in normal infants. Am J Respir Crit Care Med 2000;162:1397-402.

20 Hantos Z, Daroczy B, Suki B, et al. Input impedance and peripheral inhomogeneity of dog lungs. J Appl Physiol 1992;72:168-78.

21 Fredberg JJ, Stamenovic D. On the imperfect elasticity of lung tissue. J Appl Physiol 1989;67:2408-19.

22 Tiddens HA. Detecting early structural lung damage in cystic fibrosis. Pediatr Pulmonol 2002;34:228-31.

23 Petak F, Hall GL, Sly PD. Repeated measurements of airway and parenchymal mechanics in rats by using low-frequency oscillations. J Appl Physiol 1998;84:1680-6. 